\title{
TRATAMIENTO DEL DUELO EN SALUD MENTAL: UNA EXPERIENCIA GRUPAL
}

\section{GRIEF TREATMENT IN MENTAL HEALTH: A GROUP EXPERIENCE}

\author{
José Manuel Sanz Cruces \\ Psicólogo Interno Residente
}

\section{Isabel María García Cuenca \\ Psicóloga Interna Residente}

\section{Eva Carbajo Álvarez}

Jefa Unidad de Psicología Clínica y de la Salud

Consorcio Hospital General Universitario de Valencia

Cómo Referenciar este artículo/How to reference this article:

Sanz, J. M., García, I. M. y Carbajo, E. (2014). Tratamiento del duelo en Salud Mental: Una experiencia grupal. Revista de Psicoterapia, 25(99), 115-133.

\begin{abstract}
Resumen
INTRODUCCIÓN: Debido a diferentes cambios sociales, los servicios de Atención Primaria (AP) y Salud Mental (USM) son espacios en los que el paciente busca atención y alivio al dolor del duelo que no encuentra en su red social.

MATERIAL Y MÉTODO: Se realizó un grupo de apoyo al duelo de 12 sesiones con frecuencia quincenal basado en los postulados de Worden (2010) en una USM. Los participantes fueron cinco mujeres derivadas de AP y USM que conformaron un grupo heterogéneo en cuanto a gravedad, tipo y cronología de la pérdida. Se les evaluó antes $y$ después del tratamiento mediante escalas psicométricas: Entrevista Diagnóstica de Duelo Complicado (Prigerson y Jacobs, 2001), la Escala Hospitalaria de Ansiedad Depresión (HAD) (Zigmond y Snaith, 1983) y la Escala de Sueño (MOS) (Hays y Stewart, 1996) y con una encuesta de satisfacción y valoración de los resultados. RESULTADOS: No hubo resultados significativos en las escalas psicométricas y si altas puntuaciones en satisfacción y valoración. Se concluye que las pacientes perciben un alivio en su sufrimiento que parece estar relacionado con el desahogo emocional más que con la realización de cambios o la resolución de tareas del duelo. Se describen pacientes e intervenciones realizadas así como las limitaciones observadas.

Palabras clave: Duelo, terapia de grupo, tareas duelo, satisfacción, apoyo emocional.
\end{abstract}

Fecha de recepción: 11/09/2014. Fecha de aceptación: 27/10/2014.

Correspondencia sobre este artículo:

E-mail: josesanzpsicologiaclinica@gmail.com

Dirección postal: Avenida Tres Cruces, 2, 46014. Valencia. 963131800

(C) 2014 Revista de Psicoterapia 


\begin{abstract}
INTRODUCTION: Due to different social changes, Primary Health Care (PHC) and Mental Health (MH) services have became spaces in which the patient seeks the attention and the relief to the pain of mourning that cannot find in his familiar and social network.

MATERIAL AND METHOD: It took place a support group of mourning carried out in 12 sessions and based on the Worden postulates (2010). The participants were 5 women referred from $\mathrm{PHC}$ and $\mathrm{MH}$ services that formed an heterogeneous group regarding the severity, the kind and the chronology of the loss.

The participants have been evaluated before and after treatment with the following psychometric scales: Diagnostic Interview of Complicated Grief by Prigerson and Jacobs (2001), the Hospital Anxiety and Depression Scale (HADS) by Zigmond and Snaith (1983), the Medical Outcomes Stydy (MOS) by Hays and Stewart (1996) and a satisfaction survey and evaluation of the results.

RESULTS: There were no significant results in the psychometric scales but high scores in satisfaction and evaluation of the therapy. It is concluded that the patients perceive a relief in their suffering that seems to be related to emotional relief rather than with a change to face the mourning. Patients and interventions carried out have been described, as well as limitations observed.
\end{abstract}

Keywords: Grief, group therapy, tasks ofmourning, satisfaction, emotional support. 
En el Sistema Sanitario es habitual encontrar pacientes cuyo motivo de consulta son malestares asociados a procesos de duelo. Se estima que más del $25 \%$ de las demandas que se cursan en atención primaria relacionadas con aspectos psicológicos tienen su origen en la pérdida de un ser querido (Limonero, Lacasta, García, Maté y Prigerson, 2009). Esto ocurre, por un lado, por la destrucción de espacios y rituales sociales en los que expresar sentimientos y recibir apoyos y, por otro, por la consideración del sufrimiento como algo antinatural que ha de ser tratado médica y/o psicológicamente. Así, los servicios de Salud Mental (USM) y Atención Primaria (AP) se conforman como los nuevos espacios en los que el paciente busca atención y alivio a su dolor (Servicio Andaluz de Salud, 2011).

En esta circunstancia, las políticas sanitarias se refieren a la importancia de la prevención y la promoción de la salud mental para detectar complicaciones en los procesos de duelo y reducir la medicalización de problemas no psicopatológicos como la desesperación o la angustia producida por un duelo normal (Ministerio de Sanidad y Consumo, 2011).

La evidencia y los expertos concluyen que los antidepresivos son eficaces para reducir los síntomas asociados al duelo como la depresión pero no el duelo en sí mismo (Forte, Hill, Pazder, y Feudtner, 2004). En cuanto a la psicoterapia (individual, grupal, etc.), del metaanálisis de Allumbaugh y Hoyt (1999) se concluye que las diferentes modalidades de psicoterapia tienen tamaños del efecto pequeños y que los mejores resultados se obtienen cuando el doliente demanda ayuda y cuando la intervención se realiza a los pocos meses de la pérdida. Von Fortner (1999) concluye que la terapia no se justifica en personas con duelo normal pero si en personas con duelos de riesgo o complicados. En el metaanálisis de Wittouck, Van Autreve, De Jaegere, Portzky y Van Heeringen (2011), se encontró que la terapia parecían ser eficaz en el alivio a corto y largo plazo de los síntomas del duelo complicado, aumentando su efecto significativamente con el tiempo. Schut y Stroebe (2006) realiza una revisión bibliográfica sobre terapias en el duelo y concluye que las intervenciones son más efectivas cuando se aplican a duelos patológicos siendo desaconsejable intervenciones tempranas. En cuanto a la eficacia de los grupos de terapia, destaca el meta-análisis realizado por Kato y Mann (1999). De los ocho trabajos que estos autores revisan, sólo uno arroja resultados claramente positivos y en seis de ellos la intervención grupal no tiene ningún efecto. En nuestro entorno, Diaz (2011) lleva a cabo un tratamiento grupal en pacientes con duelo complicado obteniendo mejoras significativas tras la intervención.

En nuestra experiencia nos encontrábamos en la USM con un número relevante de pacientes demandantes de tratamiento para procesos de duelo normal y también complicado. Para hacer frente a esta demanda, se optó por una terapia de apoyo grupal. Las ventajas de la intervención de apoyo grupal en duelo estriban, por un lado, en parámetros de eficiencia, como el mejor aprovechamiento de los recursos o el aumento de la frecuencia de las sesiones y, por otro lado, proporcionan un espacio único para el encuentro, la reducción de los sentimientos de soledad y 
la expresión emocional de los deudos.

De esta forma, se realizó un grupo con formato cerrado y limitado en el tiempo (12 sesiones de periodicidad quincenal) inspirado en el modelo teórico de Worden (2010) de tareas del duelo. Se eligió este modelo por su reconocimiento y aceptación como modelo teórico válido (Yoffe, 2013), además de por su sencillezy aplicabilidad en la psicoeducación. Worden propone que la persona ha de realizar, de forma consciente o inconsciente, una serie de tareas para la superación del duelo: aceptar la realidad de la pérdida, reconocer y reflexionar las emociones asociadas, adaptarse a un medio en el que el fallecido está ausente y recolocar emocionalmente al fallecido. Este planteamiento exige por parte del doliente un posicionamiento activo y voluntad de superación.

Los objetivos globales de la intervención fueron principalmente dos, primero, promover un espacio de seguridad y confianza en el que los pacientes pudieran sentirse acompañados y reflexionar sobre su proceso de duelo. Segundo, impartir una psicoeducación que permitiera a los pacientes entender mejor el proceso del duelo y a sí mismos y posicionarse como agentes activos en el afrontamiento de las tareas del duelo. El objetivo del estudio fue proponer un tratamiento piloto en nuestra USM que sirviera de experiencia para futuras intervenciones grupales en duelo complicado.

\section{Material y método}

\section{Participantes}

El estudio se realizó en una USM perteneciente a la localidad valenciana de Torrente. Se ofreció tratamiento grupal sustitutivo de la terapia individual, con posibilidad de retomar las terapias individuales una vez finalizado el tratamiento, a 12 pacientes de la USM y a 6 pacientes derivados de AP. Se seleccionaron estos pacientes atendiendo a los criterios de inclusión y exclusión que se enumeran en la tabla 1. De estos 18 pacientes, tras la evaluación psicométrica y firma del consentimiento informado, 8 aceptaron iniciar el tratamiento.

\section{Tabla 1}

Criterios de inclusión y exclusión.

\begin{tabular}{cc}
\hline Criterios inclusión & Criterios exclusión \\
\hline - Duelo como motivo de consulta principal & - Comorbilidad con un trastorno \\
& psicótico, de personalidad o bipolar \\
& - Menor de edad \\
& - Consumo tóxicos \\
\hline
\end{tabular}

En la primera sesión del tratamiento se produjeron tres bajas por diferentes motivos: ruptura de expectativas, enfermedad y por mutuo conocimiento previo. Finalmente se consolidó un grupo estable de cinco mujeres que resultó ser 
heterogéneo en cuanto a edad, relación de parentesco con el difunto, tiempo desde la muerte y gravedad.

Al finalizar el tratamiento, se dieron tres altas y las dos pacientes restantes se reincorporaron a su tratamiento individual.

La evolución de la muestra viene detallada en la figura 1.

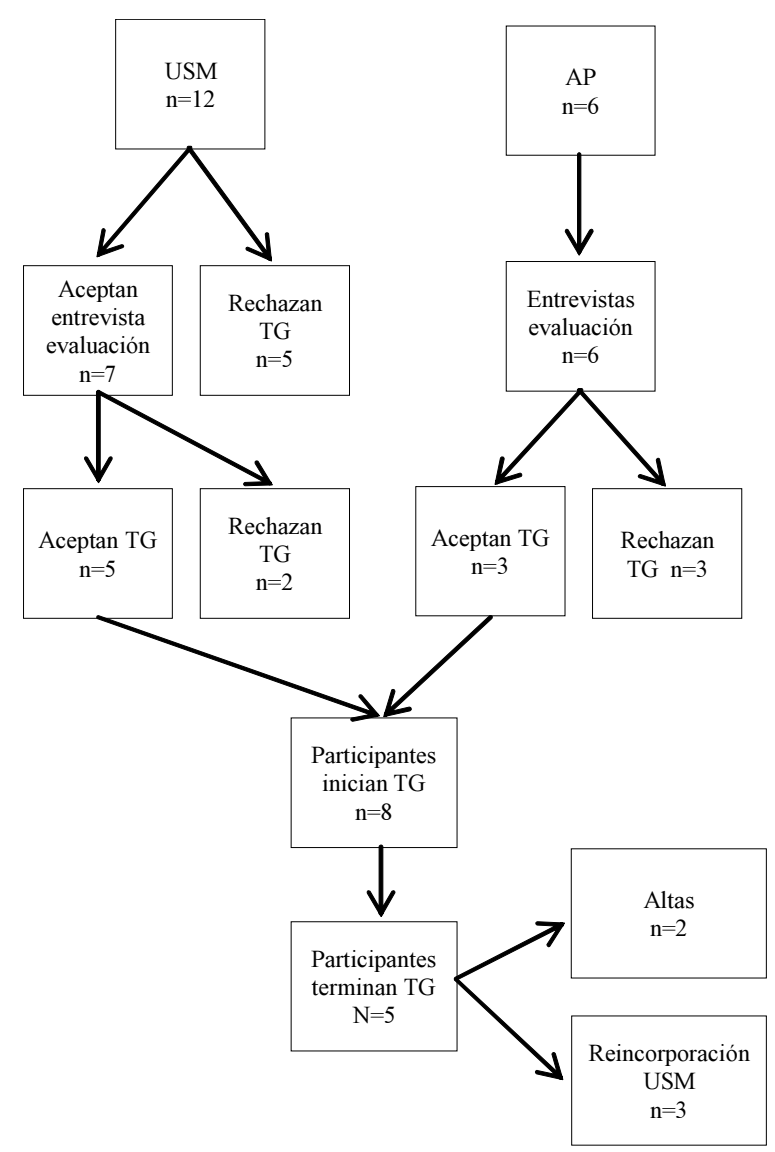

\section{Figura 1}

\section{Evolución de la muestra}

A continuación se describen los participantes que completaron la terapia:

C: Mujer de 70 años, viuda desde hace más de una década. Su hijo mayor, casado y con hijos, fallece hace siete meses debido al cáncer. Vive con su hijo diagnosticado de trastorno bipolar que se ha mudado temporalmente a vivir con ella. Acude a terapia individual en USM desde hace do meses y está en tratamiento farmacológico. La paciente se encuentra al inicio del proceso del duelo y su llanto y relato están cargados de desesperanza, sentimientos de vacío y pérdida de sentido vital. La motivación de C para acudir a terapia grupal es la ventilación emocional 
y encontrar a otras personas a las que se les hubiera muerto un hijo, circunstancia en la que parece sentirse muy sola. La paciente comenta en numerosas ocasiones "yo he perdido a mi marido y a mi madre pero perder un hijo es mucho más doloroso". Se considera un proceso de duelo normal con factores de riesgo.

M: Mujer de 28 años, menor de dos hermanos, con pareja y sin hijos. En situación de desempleo, vive con su padre. Hace ocho años fallece su madre después de padecer cáncer. En tratamiento psicológico y farmacológico en la USM desde hace tres años debido a miedo fóbico a que a sus seres queridos les pase una desgracia. La preocupación constante y conductas comprobatorias y evitativas son interpretadas como un intento ineficaz de defenderse de su sensación de falta de control sobre la muerte. La paciente padece un duelo prolongado en el que subyace un estilo de vinculación fusional con la madre ("tranquila que somos una" le dijo aquella momentos antes de morir) que dificulta la aceptación de la pérdida ya que esto supondría aceptar también la muerte de una parte muy importante de sí misma. Además, siente como una traición y una separación definitiva continuar con su vida, idea inspirada por el ejemplo de su propia madre, la cual, ante la muerte de su madre "dejó de celebrar la navidad".

$\mathrm{N}$ : Mujer de 40 años, viuda, tiene un hijo de ocho años, trabaja en un negocio familiar. Hace dos años falleció su marido de un infarto. Esto ocurrió unas horas después de una fuerte discusión en torno a la desidia de él en el cumplimiento del tratamiento para sus problemas de corazón. Durante esta discusión la paciente utiliza palabras y formas de las que en la actualidad se siente culpable. Acude a terapia debido a dificultades en una nueva relación de pareja. Esta nueva pareja se comporta dejando su propia felicidad y salvación en manos de ella, recreándose en él, el mismo estilo dependiente y pasivo de su difunto marido. Esta relación genera sentimientos de culpa y confusiones relacionadas con aspectos del duelo no elaborados. No toma fármacos. No cumple criterios duelo complicado.

S: Mujer de 60 años, viuda sin hijos ni familia. Ama de casa. Con importantes afectaciones cardíacas. En tratamiento psicológico y farmacológico en USM desde hace siete meses. Hace año y medio la paciente pierde a su marido ("la única familia que tenía"), tras varios años padeciendo un cáncer. Durante el proceso de enfermedad, la paciente se muestra muy evitativa y poco aceptante, de forma que rehúye la información médica y niega hasta el final la posibilidad del fallecimiento de su esposo. La paciente acude a terapia buscando apoyo emocional y compañía ante su tristeza y desespero. Se siente incapaz de aceptar la muerte de su marido ("yo creo que va entrar por la puerta de casa en cualquier momento"). Realiza conductas evitativas de su dolor y soledad, como momificar la habitación y mantener conversaciones imaginadas con el difunto en diferentes momentos del día con las que alivia su soledad.

I: Mujer de 50 años, separada hace diez años tras episodios de maltrato. Antecedentes de distimia y somatizaciones de siete años de evolución que coincide con la muerte de su padre. Hace algo más de un año, el mayor de sus cuatro hijos, 
casado y con hijos, fallece de cáncer. Lleva tres años en tratamiento psicológico y farmacológico en USM por distimia. Se trata de una paciente en la que el duelo del hijo se superpone al del padre de forma que no es capaz de elaborar ninguno de los dos duelos. Se siente aislada y excluida ("los demás parece que lo llevan mejor que yo"), relacionándose con los demás a través de la queja del duelo lo que causa aún más aislamiento e incomprensión con respecto a sus hijos y familiares.

\section{Instrumentos}

En la medición pre y post tratamiento se administraron las siguientes escalas psicométricas:

- La Entrevista Diagnóstica de Duelo Complicado (EDDC) (Prigerson y Jacobs, 2001) en su versión española (Limonero et al., 2009) que se compone de varios criterios. El Criterio A, con 4 ítems, mide estrés por la separación que conlleva la muerte: pena, añoranza, soledad, pensamientos intrusivos, etc. El Criterio B, con 29 ítems, se refiere más concretamente al estrés por el trauma que supone la muerte: dificultades de aceptación, adaptación, vacío existencial, pérdida de sentido, etc. En cuanto a las propiedades psicométricas de la versión española, los autores informan de un alfa de Cronbach de 0.88 y fiablidad test-retest de 0.81 .

- La Escala Hospitalara de Ansiedad Depresión (HAD) (Zigmond y Snaith, 1983), en su versión española (Terol et al., 2007), es un cuestionario autoaplicado de 14 ítems, integrado por dos subescalas de 7 ítems, una de ansiedad y otra de depresión. La intensidad o frecuencia del síntoma se evalúa en una escala de Likert de 4 puntos (rango 0-3). Su consistencia es alta con un alfa de Cronbach de 0.86 (ansiedad) y 0.86 (depresión).

- La Escala de Sueño (MOS) (Hays y Stewart, 1996), en su versión española (Hays, Martin, Sesti y Spritzer, 2005), proporciona información subjetiva sobre la calidad y la cantidad de sueño. La ES-MOS facilita puntuaciones en las 6 subescalas que oscilan entre 0 y 100. Su consistencia interna es de 0.73 .

Se eligieron estas mediciones por su brevedad, fiabilidad, validez y su pertinencia para detectar posibles cambios en el proceso del duelo, dado que éste suele cursar con estado de ánimo deprimido, ansiedad y dificultades para dormir (Barreto, 2012).

En la evaluación post tratamiento, sumado a los inventarios descritos, se administraron de forma anónima y grupal una encuesta ad-hoc con la que se trató de conocer la satisfacción y valoración que los pacientes hacían del tratamiento grupal.

Para ello, los sujetos debían puntuar 3 afirmaciones sobre diferentes aspectos de mejoría con un rango de 1 (muy en desacuerdo) a 5 (muy de acuerdo): a) experiencia subjetiva: "me siento mejor conmigo misma ahora que cuando empecé la terapia (ejemplo: más autoestima, menos culpa, menos soledad, etc.)", b) cambios conductuales: "la terapia me ha ayudado a hacer cambios en la forma de llevar mi vida, como tomar decisiones sobre mi duelo y mi vida y llevarlas a cabo 
(ejemplo: socializarme, hacer cambios en la casa, desprenderse de objetos del fallecido, etc.)" y c) alivio de los síntomas: "pienso que la terapia me ha ayudado a tener menos síntomas de ansiedad y depresión (ejemplo: menos dolores corporales, insomnio, pérdida de apetito, llanto, preocupación, etc.)".

En cuanto a la encuesta de satisfacción, se centró en los siguientes aspectos: a) apoyo emocional (6 preguntas). Ejemplo: "En el grupo he tenido un espacio para hablar y reflexionar sobre mi duelo", b) psicoeducación (5 preguntas). Ejemplo: "La información ofrecida por el terapeuta me ha ayudado a comprender mejor mi propio duelo", c) terapeuta (6 preguntas). Ejemplo: "Me he sentido motivada por el terapeuta para mejorar en mi duelo" y d) marco terapéutico (4 preguntas). Ejemplo: "Me hubiera gustado tener más sesiones".

Para finalizar la evaluación, de realizaron 4 preguntas abiertas (tabla 8) para conocer la opinión de los participantes sobre la experiencia. Además, se les pidió que ordenaran los componentes de la terapia según la utilidad percibida.

\section{Procedimiento}

El proyecto se inició con el proceso de selección de participantes durante el cual surgieron las primeras dificultades ya que 10 de los 18 pacientes seleccionables declinaron la terapia grupal en su mayoría por el pudor a compartir la historia de pérdida con personas extrañas.

La primera entrevista tras la derivación se realizó con aquellos sujetos que accedían voluntariamente al tratamiento grupal y cumplían los criterios de inclusión-exclusión. En esta entrevista se les administró, previa firma del consentimiento informado, las pruebas psicométricas pre tratamiento. En una segunda entrevista se realizaba el contrato terapéutico en el que se especificaba el marco de la intervención con objeto de obtener un compromiso del paciente y aumentar la adherencia al tratamiento. Al final de la última sesión se administraron de nuevo las pruebas psicométricas pretratamiento y una encuesta de satisfacción y valoración de los resultados anónima realizada ad-hoc.

El encuadre grupal se organizó en torno a las siguientes características: grupo cerrado, con una asistencia media de 4.5 pacientes por sesión, sesiones prefijadas que sumaron un total de 12 de frecuencia quincenal y hora y media de duración. El tratamiento se prolongó durante seis meses.

Las sesiones tuvieron la siguiente estructura: 1) ventilación emocional: se trataba de que las pacientes respondieran a la pregunta “¿cómo te sientes?" para que pudieran examinar su estado emocional en el aquí y ahora y expresar su malestar, 2) resumen de los conflictos y aspectos más relevantes de la sesión anterior, 3) reflexión o conversación en torno al tema propuesto que solía ser alguna de las tareas del duelo de Worden (Worden, 2010), 4) mensaje de cambio en el que el terapeuta utilizaba frases alentadoras y de búsqueda de insight y 5) ejercicios finales de relajación-mindfulness.

Los datos del estudio fueron analizados con el programa SPSS versión 19.0. 
Para explorar las diferencias entre la medición antes y después del tratamiento se utiliza la prueba no paramétrica de los rangos de Wilcoxon debido a que el tamaño de la muestra es inferior a 30 por lo que no se puede utilizar la prueba paramétrica t de Student.

En la siguiente tabla se resumen las sesiones realizadas:

Tabla 2

Resumen de los objetivos, contenidos y mensajes de cambio.

\begin{tabular}{|c|c|}
\hline Tarea y objetivos & Reflexión propuesta y comentarios \\
\hline $\begin{array}{l}\text { Sesión } 1 \\
\text { Presentación: } \\
\text { Generar clima cáli- } \\
\text { do y de acogida. } \\
\text { Ajustar expectati- } \\
\text { vas. } \\
\text { Definir contrato } \\
\text { grupal y reglas. }\end{array}$ & $\begin{array}{l}\text { Presentación de los componentes del } \\
\text { grupo mediante dinámica proyectiva: } \\
\text { los participantes han de contestar a } \\
\text { unas preguntas simples sobre gustos } \\
\text { imaginándose lo que contestaría la } \\
\text { persona del grupo que le había tocado } \\
\text { por azar. Expectativas y temores: cada } \\
\text { persona escribe qué le gustaría que } \\
\text { sucediera en el grupo y que no. Se } \\
\text { pone en común y se debate. } \\
\text { Primeros abandonos: ruptura de ex- } \\
\text { pectativas en una paciente que espe- } \\
\text { raba un grupo "para conocer gente" y } \\
\text { no un grupo de trabajo en torno al } \\
\text { duelo. }\end{array}$ \\
\hline
\end{tabular}

\section{Sesión 2}

Tarea I: Aceptar la realidad de la pérdida.

Objetivos: Indagar sobre el significado de la persona fallecida. Entender quién ha muerto.
Dinámica para la memorización de nombres: los participantes debían pasarse una "pelota de energía" imaginaria lo más rápidamente posible nombrando antes a la persona receptora. El ejercicio finaliza cuando se pasan la "pelota" entre ellos de forma fluida.

Presentar al ser querido fallecido al grupo: ¿cómo se llamaba?, ¿cuál era tu relación de parentesco?, ¿cómo era físicamente?, ¿qué cosas le gustaban?, ¿a qué se dedicaba? etc.

Indagar sobre el vínculo: ¿qué te aportaba tu ser querido? (seguridad, compañía, valía, diversión...), ¿qué se ha llevado con su muerte?, ¿qué es lo que más echas de menos de todo?

Se advierte de la posibilidad de que, al rememorar e identificarse con las historias de pérdida de los otros, sientan un empeoramiento temporal.

Mientras algunas pacientes desean hablar otras se muestran inhibidas y con
Mensaje de cambio

La elaboración adecuada del duelo requiere de un esfuerzo personal.

La negación de la pérdida es muy común en los dolientes. Se trata de un vano intento por evitar el sufrimiento a corto plazo. La negación, se puede presentar tratando de conservar todo aquello que pertenecía o recuerda al fallecido, generando la ilusión de que nada ha cambiado y evitando de este modo el punto de partida de todo duelo que es la aceptación de la dolorosa realidad. 
miedo a hacerlo. Se trata de que al menos digan el nombre de la persona difunta.

La paciente M. habla con el terapeuta en privado al final de la sesión, se cuestiona seguir, se siente avergonzada (infantilizada, inadecuada) al comparar su pérdida (madre) con el de otras pacientes (hijos). Señalamos la importancia de estos sentimientos y su adecuación al grupo.

\section{Sesión 3}

Tarea I: Aceptar la realidad de la pérdida.

Objetivos: Tomar conciencia de la pérdida relatando la historia del propio duelo, en un clima de confianza.

Relato guiado de la pérdida, a través de 3 niveles, con el objetivo de aumentar la integración y conciencia del suceso (Neimeyer, 2007):

1. La historia objetiva: “¿dónde estabas cuando te dieron la noticia?".

2. La historia enfocado a la emoción: "¿qué sentiste física y corporalmente?".

3. La historia orientada al significado:

\section{Tarea II: Trabajar las emociones y el dolor de la pérdida.}

Sesión 4

Objetivos: Ayudar a los pacientes a identificary expresar sentimientos. “¿qué entendiste?”, “¿qué pensabas?".

Se observan en algunas pacientes actitudes de negación que se remontan al proceso de enfermedad.

Las pacientes hablan sobre Dios. Sienten rabia e incomprensión "¿por qué a mí?". Señalamos que estos sentimientos podrían estar detrás de dificultades para aceptar la pérdida y dar sentido a la muerte.

\section{Hablar sobre los sentimientos y reaccio-} nes por las que se pasa en un duelo, especialmente la tristeza y la desesperación.

Durante los relatos individuales el terapeuta normaliza, identifica y valida los sentimientos que los pacientes utilizando como recurso las experiencias de otros pacientes.

La desesperación en el relato de las participantes resulta muy complicada de contener y soportar y produce dudas sobre la viabilidad del grupo.

Tarea para casa: "carta que no se envía". El objetivo de esta carta es identificar y expresar los sentimientos hacia el ser querido. Muchas veces no nos sen-
Todo duelo es el proceso normal que sigue a la pérdida de lo inmensamente querido. El duelo forma parte integral de la relación amorosa, no es el fin ni la interrupción del amor, sino una de sus fases naturales; así el duelo no interrumpe la relación, sigue siendo amor.

Es importante aceptar estos sentimientos de tristeza, desesperación y rabia, pero no invitarlos, atenderlos pero no habitar con ellos. Estos sentimientos son un huésped normal, aceptable y tolerable, pero poco saludable cuando la visita es por mucho tiempo.

Las verdaderas emociones son positivas y evolucionan en la medida en que uno las expresa. Lo que hace 
timos orgullos de estos sentimientos (culpa, rabia, miedo, pena e impotencia) pero puede ser importante tomar conciencia de dichas emociones y la forma en la que las expresamos.

que la experiencia dolorosa que supone expresar nuestras emociones sea constructiva o destructiva es lo que hacemos con ese dolor, el sentido que le damos.

Sesión 5
Tarea II: Trabajar las
emociones y el dolor
de la pérdida.
Objetivos:
Normalizar y enten-
der los miedos y la
ansiedad.

Explicitar qué miedos y angustias se tenían antes del fallecimiento y cuáles se tienen ahora.

Preparamos las navidades: los participantes hablan sobre la dificultad de estas fechas y sugerimos la idea del duelo como un proceso dinámico en el que existen momentos de avance y retroceso.

Las pacientes llevan alguna sesión sentándose en las mismas posiciones.

Se producen intentos de formar alianzas entorno al mensaje "perder a un hijo es mucho peor que a un marido" que hubo que abordar para evitar posibles sentimientos de invalidación en otros pacientes. Estas alianzas se abordaron validando el dolor e interpretando explícitamente la fantasía de fusión "¿deben sentirse muy solas en su dolor?".

Ninguna realiza la tarea para casa.

Sesión 6

Tarea II: Trabajar las emociones y el dolor de la pérdida.

Objetivos: Explorar sentimientos de enfado o culpa.

Ayudarles a que puedan pedir perdón y perdonarse.
Hablar sobre los asuntos por las que se sienten culpables. Luego han de contestar las siguientes preguntas: ¿pueden hacer algo actualmente para cambiar el pasado?, ¿se sienten perfectos o ilimitados?, ¿ha habido alguien en su vida al que hayan perdonado?, ¿por qué lo perdonaron?

La culpa gira entorno a seguir viviendo mientras la persona querida está muerta. El enfado se dirige hacia Dios, los médicos y los demás.

Las pacientes hablan de amigos y familiares. Por un lado se sienten vacías y rechazadas ("una carga") y temen acercarse. Por otro lado, rabiosas e incomprendidas ("no saben lo que se sufre") y las gustaría que los demás se ocuparan de ellas. Se tratan las dificultades y limitaciones de los demás para
Cuando experimentamos una muerte cercana todos los miedos y todas las aprehensiones surgen a la vez en el intento de protegernos de futuras desgracias. Tratamos de afrontar este miedo transformándolo en evitaciones, controles, prohibiciones o sobreprotección. Al actuar esta sensación de falta de control y ansiedad, se potencia y alimenta a su vez la angustia y el miedo

La culpa surge a partir de la presuposición errónea de que somos perfectos y por lo tanto no podemos cometer errores y de que somos ilimitados y podemos hacerlo todo bien siempre, e incluso, cambiar el pasado.

Aceptando nuestra condición imperfecta se puede ir mitigando la culpa. 
entender y la falta de un espacio fuera del grupo para hablar del duelo.

Se quedan hablando en la sala tras la sesión.

Sesión 7

Tarea III: Adaptarse a un medio en el que el fallecido está ausente.

Objetivos: Replantear nuevos roles $y$ actividades.
Tratar de que identifiquen las dificultades en su vida cotidiana más allá del dolor sentimental como; la pérdida de rol, la pérdida de apoyo, la pérdida de sentido de la vida, la soledad o las pequeñas dificultades de la vida cotidiana sin la persona querida.

Pensamientos rígidos y entorno al papel de las demás personas: "los demás tiene que darse cuenta de que estoy mal". Este pensamiento genera rabia, aislamiento y dificulta pedir ayuda. Se trata de que conecten con su deseo de estar acompañadas y los recursos que tienen para realizarlo.

Tarea para casa: Pensar en pequeños objetivos a realizar durante los próximos días.

\section{Sesión 8}

Tarea III: Adaptarse a un medio en el que el fallecido está ausente.

Objetivos: Ayudar a que los pacientes se abran a la idea del enriquecimiento espiritual a través de la experiencia de la muerte y a que encuentren un nuevo sentido en sus vidas.
Tratar el tema de la pérdida de sentido tras la muerte del ser querido y la importancia de encontrar uno nuevo: ¿Han cambiado sus valores después de la pérdida?, ¿cuáles era antes las cosas importantes para vosotros y cuales lo son ahora?, ¿qué sentido va a tener esa pérdida en su vida?, ¿qué cambios prevén en el conocimiento de sí mismo y en la orientación de sus vidas?, ¿qué nuevos recursos han encontrado en sí mismos después de la muerte del ser querido?

Las pacientes no son capaces de proyectarse al futuro e imaginar una mejoría. Tampoco de articular un significado o valor personal ante la experiencia de la muerte.

Ninguna realiza la tarea para casa.
Aunque el espacio que ocupaba la persona querida no nunca se podrá llenar, si es posible volver a abrirse a la experiencia en el mundo de los vivos y posicionarse de una forma responsable con el propio bienestar. Esta reinvención de uno mismo es, al fin y al cabo, lo que la persona amada hubiera querido para nosotros.

No volverán a ser las mismas personas que antes de la pérdida, pero obtendrán la riqueza emocional y la profundidad que proviene de haber conocido el dolor intenso y también su curación.

\section{Sesión 9}

Tarea III: Adaptarse a un medio en el que el fallecido está ausente

Objetivos:Ayudar a que los pacientes cie-
Abordar la cuestión de los asuntos inconclusos: ¿Qué cosas les hubiera gustado decir que no hicieron?, ¿qué cosas dijeron o hicieron de las que se arrepienten?, ¿qué acciones reparadoras podrían realizar en la actualidad para tratar de subsanar las cosas que no hicieron o
Cuando quedan asuntos inconclusos se hace muy difícil la resolución adecuada del duelo. Es importante entonces ir resolviendo dichos asuntos para poder 
rren asuntos incon- no dijeron o las cosas de las que se clusos.

arrepienten?
Lo más comentado fueron aquellos sentimientos positivos que nunca pudieron expresar.

\section{Sesión 10}

Tarea IV: Recolocar emocionalmente al fallecido y continuar viviendo

Objetivos:Animar al paciente a decir un "adiós temporal".
Con ayuda de la foto decir unas palabras de despedida.

En esta sesión surgieron profundas resistencias que se manifestaron en forma de incomprensión hacia el motivo del ejercicio y comentarios implícitos sobre la incapacidad del terapeuta para entender su circunstancia de dolor emocional.

gradualmente despedirse del fallecido.

Para poder dedicar energía a los vínculos e intereses que les esperan en la vida, es muy recomendable poder desplazar la atención de la persona fallecida al mundo de los vivos. Para ello, puede serútil dedicar un tiempo a despedirse gradualmente y recolocar emocionalmente al difunto.

\section{Sesión 11 \\ Tarea IV: Recolocar emocionalmente al fallecido y continuar viviendo}

Objetivo:Entender el significado de despedirse
Se trabaja la idea de que ellas merecen ser felices y se explora el significado de despedirse.

Con la finalidad de comprender el por qué de la resistencia de la sesión anterior y encontrar las razones que bloqueaban el cambio, se preguntó a los pacientes que significa para ellos despedirse de la persona querida. Entre otras cuestiones surgieron temores a sentirse aún más deprimidos y solos, a olvidar o a ser traidores. Esta intervención redujo la resistencia y explicitó unos conflictos susceptibles de tratamiento.
Seguir adelante en la vida con los demás vivos no significa olvidar al fallecido si no integrar dentro de uno y darle un nuevo sentido o forma a la relación que tenemos con el difunto.

Mantenerse triste y deprimido durante un largo tempo no es una prueba de amor.

\section{Sesión 12}

Tarea IV: Recolocar emocionalmente al fallecido y continuar viviendo

Objetivos:Despedida del grupo.

Expresión de las opiniones sobre el tratamiento.

Incentivarlas a la adquisición de nuevos vínculos e intereses.
Que escriban y compartan con el grupo

cuáles serían sus planes o metas a corto, medio y largo plazo y cuáles serían sus nuevos vínculos e intereses.

Algunos pacientes muestran su deseo de continuar con la terapia o de haber tenido alguna sesión más.

Se intercambian teléfonos y direcciones al final de la terapia.
Han aprendido que no siempre tienen el control de las circunstancias vitales. Deben usar este descubrimiento para vivir más intensamente, para disfrutar más a cada momento. Deben continuar manteniendo razones para vivir. Tienen un futuro por el que vale la pena resistir y merecen encontrar un nuevo sentido de propósito y placer en la vida. 


\section{Resultados}

\section{Datos de los participantes}

En la tabla 3 se presentan los datos sociodemográficos, los datos sobre el duelo y de tratamiento previo en la USM.

\section{Tabla 3.}

Datos sociodemográficos, tipo de duelo y tratamiento previo

\begin{tabular}{ll}
\hline Sexo & \\
• Mujer/Hombre & $5 / 0$ \\
\hline Edad & \\
• Media, min-máx. & $50.4,27-70$ \\
\hline Hijos & $3 / 2$ \\
\hline Estado civil & \\
• Casada o en pareja & 1 \\
- Divorciada & 1 \\
- Viuda & 3 \\
\hline Tipo de convivencia & \\
- Sólo & 1 \\
- Hogar familiar & 3 \\
- Con sus hijos & 1 \\
\hline Ocupación & \\
• Labores domésticas & 2 \\
- Desempleo & 1 \\
• Activo & 1 \\
• Jubilado & 1 \\
\hline Estudios & 1 \\
• Leer y escribir & 4 \\
- Primarios & 1 \\
\hline Secundarios &
\end{tabular}

Relación de con el fallecido

- Madre

2

- Esposa

- Hija 1

Temporalidad de la muerte

- Gradual

- Repentina

4

1

Causa de la muerte

- Enfermedad 5

- Traumatismo 0

Tiempo desde la muerte (en meses)

- Media, min-máx. $29.4,7-96$ 


\begin{tabular}{ll}
\hline $\begin{array}{l}\text { Diagnóstico duelo complicado } \\
\text { - Si/No }\end{array}$ & $3 / 2$ \\
\hline $\begin{array}{l}\text { En tratamiento psicológico } \\
\quad \text { Si/No }\end{array}$ & $4 / 1$ \\
\hline $\begin{array}{l}\text { Duración tratamiento psicológico (meses) } \\
\quad \text { Media, min-máx. }\end{array}$ & $20.25,2-36$ \\
\hline $\begin{array}{l}\text { En tratamiento con antidepresivos } \\
\quad \text { Si/No }\end{array}$ & $3 / 2$ \\
\hline $\begin{array}{l}\text { Duración tratamiento antidepresivos (meses) } \\
\quad \text { Media, min-máx. }\end{array}$ & $34.4,7-84$ \\
\hline
\end{tabular}

\section{Pruebas psicométricas pre-post intervención}

En la tabla 4 se prese ntan los estadísticos descriptivos de la sintomatología ansioso-depresiva, calidad del sueño y criterios A y B de la EDDC antes y después del tratamiento de los cinco sujetos que completaron el tratamiento.

Tabla 4.

Estadísticos Descriptivos, $n=5$

\begin{tabular}{lcccccccccccc}
\hline & \multicolumn{3}{c}{ HAD } & \multicolumn{3}{c}{ MOS } & \multicolumn{4}{c}{ EDDC } \\
& \multicolumn{1}{c}{ Ansiedad } & Depresión & & & \multicolumn{3}{c}{ Criterio A } & \multicolumn{2}{c}{ Criterio B } \\
\hline & Pre & Post & Pre & Post & Pre & Post & Pre & Post & Pre & Post \\
\hline Media & 12,0 & 10,4 & 13,6 & 12,4 & 52,8 & 48,8 & 10,6 & 12,2 & 90,6 & 100,6 \\
\hline D.T. & 5,87 & 3,84 & 7,02 & 4,04 & 4,02 & 5,31 & 3,28 & 4,65 & 29,34 & 34,63 \\
\hline
\end{tabular}

En la tabla 5 se muestra la diferencia de medias entre el momento pre y el post tratamiento para los mismos sujetos.

Tabla 5

Estadísticos de contraste, $n=5$.

\begin{tabular}{lccccc}
\hline & \multicolumn{2}{c}{ HAD } & MOS & \multicolumn{2}{c}{ EDDC } \\
\cline { 2 - 6 } & $\begin{array}{l}\text { Ansiedad } \\
\text { Pre-Post }\end{array}$ & $\begin{array}{c}\text { Depresión } \\
\text { Pre-Post }\end{array}$ & Pre-Post & $\begin{array}{c}\text { Criterio A Criterio B } \\
\text { Pre-Post }\end{array}$ & Pre-Post \\
\hline $\mathbf{Z}$ & $-1,09$ & $-0,68$ & $-1,36$ &, 00 &, 00 \\
\hline Sig. asintót. (bilateral) & 0,27 & 0,5 & 0,17 & 1,00 & 1,00 \\
\hline Nota. Prueba de los rangos con signos de Wilcoxon. $\mathrm{n}=5$ & & \\
\hline
\end{tabular}

\section{Valoración subjetiva de los resultados}

En la tabla 6 se presenta la valoración subjetiva de los participantes en diferentes aspectos. 


\begin{tabular}{lccc}
$\begin{array}{l}\text { Tabla } 6 \\
\text { Valoración del resultado del tratamiento, } \boldsymbol{n = 5}\end{array}$ & $\begin{array}{c}\text { Experiencia } \\
\text { subjetiva }\end{array}$ & $\begin{array}{c}\text { Cambios } \\
\text { conductuales }\end{array}$ & $\begin{array}{c}\text { Alivio de los } \\
\text { síntomas }\end{array}$ \\
\hline Media & 4,40 & 1,80 & 3,80 \\
\hline D.T. & 0,55 & 1,30 & 0,84
\end{tabular}

Nota. Puntuación de 1 a 5

En la tabla 7 se indica la satisfacción post intervención.

Tabla 7

Satisfacción, $n=5$

\begin{tabular}{lcccc}
\hline & $\begin{array}{c}\text { Satisfacción apoyo } \\
\text { emocional }\end{array}$ & $\begin{array}{c}\text { Satisfacción } \\
\text { psicoeducación }\end{array}$ & $\begin{array}{c}\text { Satisfacción } \\
\text { terapeuta }\end{array}$ & $\begin{array}{c}\text { Satisfacción } \\
\text { marco }\end{array}$ \\
\hline Media & 4,73 & 4,96 & 5 & 3,06 \\
\hline D.T. & 0,38 & 0,09 & 0,00 & 1,04 \\
\hline
\end{tabular}

En la tabla 8 se expone las contestaciones literales escritas por los sujetos a las preguntas abiertas realizadas.

Tabla 8

Preguntas abiertas y contestaciones agrupadas, $n=5$

\begin{tabular}{ll}
\hline Preguntas & Contestaciones \\
\hline “¿Qué es lo que mejor & $\begin{array}{l}\text { Apoyo o soporte emocional por parte del grupo y el } \\
\text { valoras de la }\end{array}$ \\
$\begin{array}{l}\text { experiencia } \\
\text { terapéutica?” }\end{array}$ & $\begin{array}{l}\text { Expresión de los afectos } \\
\text { Sensación de no estar sola }\end{array}$ \\
\hline “¿Y lo que peor?” & Relato de la pérdida \\
& Sesión en la que se trató la despedida \\
\hline “¿Qué quitarías o & Nada \\
cambiarias? & \\
\hline “QQué añadirías?” & Más sesiones \\
\hline
\end{tabular}

Por último, se pidió a los sujetos que ordenaran de más útil a menos útil (de 1 a 7) los diferentes componentes de la terapia. Se presentan los componentes según el orden medio asignado $(\mathrm{n}=5)$ :

1. Ventilación emocional (media: 1,2).

2. Apoyo o soporte del grupo (media: 2,2).

3. Apoyo o soporte del terapeuta (media: 3 ).

4. Reflexión grupal o conversación entorno la temática propuesta (media: 4,4).

5. La información y los mensajes de cambio (media: 5,2 ). 
6. Relajación (media: 5,2 ).

7. Tareas para casa (media: 6,8 ).

\section{Altas y reincorporaciones a la USM}

Al finalizar el tratamiento grupal se pacta el alta con tres de las cinco pacientes: $\mathrm{M}, \mathrm{N}$ y $\mathrm{C}$ por mejoría, mientras que $\mathrm{S}$ e I se reincorporan a sus tratamientos individuales.

\section{Discusión}

Los resultados de las pruebas psicométricas muestran una disminución consistente pero no significativa de la sintomatología ansioso-depresiva y de las dificultades a la hora de dormir. Por el contrario, las puntuaciones de los criterios A y B de la EDDC (Prigerson y Jacobs, 2001), aumentan sin llegar tampoco a la significación. Estos resultados, en los que no se aprecia respuesta al tratamiento, coincidiría con las conclusiones del metanálisis realizado por Kato y Mann (1999) que concluyen que la terapia grupal no resulta eficaz para aliviar el sufrimiento del doliente.

En cuanto a los resultados de la encuesta de valoración de los resultados, las pacientes aprecian mejoría en su experiencia subjetiva (al reducirse su soledad, su estigma y aumentar su autoestima) y sienten un moderado alivio de la sintomatología ansiosa-depresiva, aunque la terapia no les ha ayudado a tomar decisiones o a realizar cambios conductuales con respecto a su proceso de duelo. Además se muestran muy satisfechas con la terapia, a excepción del número de sesiones que hubieran preferido aumentar.

Estos resultados podrían evidenciar limitaciones de la terapia grupal como las descritas en Pérez et al. (2000), entre las que destacamos la socialización alrededor del dolor que fomenta el inmovilismo y perpetuación en el papel de doliente. Además existieron dificultades a la hora de adaptar el enfoque de tareas del duelo al formato grupal debido a la heterogeneidad de tareas por resolver en los participantes. Esto produjo que el tratamiento se dispersara en diferentes objetivos sin llegar a profundizar. También hay que tener en cuenta la complejidad y dificultad del proceso de duelo. El terapeuta necesita entender que la sintomatología de los pacientes, como la necesidad de negar la muerte y aferrarse al fallecido, responde a la mejor manera que el paciente ha encontrado para hacer frente a la intensa soledad, la profunda tristeza, el miedo a traicionar o a olvidar al ser amado o la toma de conciencia de la muerte. Este intento de solución, al cronificarse, puede convertirse en una forma de vinculación con los demás y la realidad muy resistente al proceso terapéutico (Mackinnon et al., 2008).

No obstante, a pesar de las dificultades expuestas, se generó un grupo cohesionado, atendiendo a la definición de Vinogradov y Yalom (1996) en el que las pacientes encontraban apoyo y podían expresar, sin miedo a la crítica, su soledad y sufrimientos y en el que se trabajó el concepto de duelo como un proceso a elaborar 
mandando un mensaje desculpabilizante a las pacientes de esperanza y responsabilidad con sus propias vidas.

Tras la experiencia se podría sugerir para futuros tratamientos grupales: 1) excluir de la terapia personas con duelos normales y sin factores de riesgo, 2) formar grupos más homogéneos en cuanto a la gravedad, tipo y cronología de la pérdida para favorecer la cohesión y focalización del tratamiento, 3) incluir un cooterapeuta que ayude en la contención de las emociones y en el señalamiento de resistencias, 4) fomentar desde el inicio la conciencia del problema que no es tanto el sufrimiento y las emociones desgarradoras que acompañan al duelo como la dificultad de integrar la experiencia de la muerte y afrontar o dar significado a una nueva vida sin la persona amada, 5) comprender y aceptar en fases tempranas de la terapia los motivos de los pacientes para resistirse al cambio a fin de reducir la frustración en el terapeuta y guiar la intervención.

Como conclusión, de los datos recogidos por las evaluaciones psicométricas y cualitativas, se desprende que la terapia grupal parece no haber sido efectiva para motivar un cambio o facilitar la elaboración del duelo. No obstante, las pacientes sienten un alivio en su sufrimiento que parece estar relacionada con la psicoeducación y el desahogo emocional brindado por el grupo y el terapeuta, más que por la realización de cambios en sus vidas.

\section{Referencias bibliográficas}

Allumbaugh, D.L. \& Hoyt W. (1999). Effectiveness of grief therapy: A meta-analysis. Journal Counselling of Psychology, 46 (3) 70-80.

Barreto, P., De la Torre O., \& Pérez-Martín M. (2012). Detección de duelo complicado. Psicooncología, 9 (2-3), 355-368. Recuperado de: http://revistas.ucm.es/index.php/PSIC/article/viewFile/40902/39159

Díaz, J. (2011). Estudio de variables asociadas a la psicoterapia grupal en los procesos de duelo patológico. Revista de la Asociación Española Neuropsiquiatría, 31 (109), 93-107. Recuperado de http://www.revistaaen.es/ index.php/aen/article/view/16120

Forte, A.L., Hill, M., Pazder, R. \& Feudtner, C. (2004) Bereavement care interventions: a systematic review. BMC Palliative Care, 3(3). Recuperado de http://www.ncbi.nlm.nih.gov/pubmed/15274744\#

Hays, R.D. \& Stewart, A.L. (1996). Sleep Measures. En Stewart, A.L., Ware, J.E. (Eds.), Measuring functioning and well-being. The Medical Outcomes Study approach (pp. 235-259). Durham y Londres: Duke University Press.

Hays, R.D., Martin, S.A., Sesti, A.M. \& Spritzer, K.L. (2005). Psychometric properties of the medical outcomes study sleep measure. Sleep Medicine, 6, 41-44. Recuperado de http://www.ncbi.nlm.nih.gov/pubmed/ 15680294

Kato, P.M. \& Mann, T. (1999). A synthesis of psychological interventions for the bereaved. Clinical Psychology Review, 19 (3), 275-296. Recuperado de http://www.ncbi.nlm.nih.gov/pubmed/10097872

Limonero, J. T., Lacasta, M., García, J.A., Maté, J. \& Prigerson, H.G. (2009). Adaptación al castellano del inventario de duelo complicado. Medicina paliativa, 16(5), 291-297. Recuperado de http://www.secpal.com/ medicina_paliativa/index.php?acc $=$ verart\&idart $=521$

Mackinnon, R.A., Michels, R. \& Buckley, P.J. (2008). La entrevista psiquiátrica en la práctica clínica. Barcelona: Ars Medica.

Ministerio de Sanidad y Consumo. (2011). Estrategia en Salud mental del Sistema nacional de salud 2009-2013. Madrid: Ministerio de Sanidad y Consumo.

Neimeyer, R. (2007). Aprender de la pérdida: una guía para afrontar el duelo. Barcelona: Paidós. 
Pérez, P., Hernangómez, L. \& Santiago, C. (2000). Terapia de grupo en duelo complicado: un enfoque desde los factores de cambio positivo. Psiquiatría Pública,12 (3), 273-284. Recuperado de http://www.aen.es/ index.php?option $=$ com_docman\&task $=$ doc_details\&gid $=406 \&$ Itemid $=52$

Prigerson, H.G. \& Jacobs, S.C. (2001). Traumatic Grief as a distinct disorder: a rationale, consensus criteria, and empirical test. En M.S. Stroebe, R.O. Hansson, W. Stroebey H. Schut (Eds.), Handbook of Bereavement Research: Consequences, coping, and care (pp. 613-48). Washington DC: American Psychological Association.

Schut, H. \& Stroebe, M.S. (2006). Interventions to enhance adaptation to bereavement: A review of efficacy studies. Journal of Palliative Medicine, 8 (1), 140-147. Recuperado de http://www.ncbi.nlm.nih.gov/pubmed/ 1649946

Servicio Andaluz de Salud (2011). Guía para profesionales de la salud ante situaciones de duelo. Andalucía: Área de Evaluación y Calidad. Escuela Andaluza de Salud Pública. Recuperado de http://www.juntadeandalucia.es/ servicioandaluzdesalud/publicaciones/listadodeterminado.asp?idp $=479$

Terol, M.C., López-Roig, S., Rodríguez-Marín, J., Martí-Aragón, M., Pastor, M. A. \& Reig, M.T. (2007). Propiedades psicométricas de la escala hospitalaria de ansiedad y depresión (HAD) en población española. Ansiedad y Estrés, 13 (2-3), 163-176.

Vinogradov, S. \& Yalom, I. (1996). Guía breve de psicoterapia de grupo. Barcelona: Paidós.

Von Fortner, B. (1999). The effectiveness of grief counseling and therapy: a quantitative review. Tesis doctoral no publicada, Memphis University, USA.

Wittouck, C., Van Autreve, S., De Jaegere, E., Portzky, G. \& Van Heeringen, K. (2011). The prevention and treatment of complicated grief: A meta-analysis. Clinical Psychology Review, 31, 69-78. Recuperado de http://www.ncbi.nlm.nih.gov/pubmed/21130937

Worden, J.W. (2010). El Tratamiento del duelo: asesoramiento psicológico y terapia. Barcelona: Paidós.

Yoffe, L. (2013). Nuevas concepciones sobre los duelos por pérdida de seres queridos. Avances en Psicología, 21(2), 129-143. Recuperado de http://www.unife.edu.pe/publicaciones/revistas/psicologia/2013/2/ Laura\%20-Yoffe.pdf

Zigmond, A. \& Snaith, R. (1983). The Hospital Anxiety and Depression Scale. Acta Psychiatrica Scandinavica, 67, 361-370. Recuperado de http://www.ncbi.nlm.nih.gov/pubmed/68808 